\title{
Aéroportie
}

\section{Hepatic portal venous gas}

\section{Tesnière $\cdot$ L. Marie $\cdot$ P. Lesage}

Reçu le 15 octobre 2012 ; accepté le 15 novembre 2012

(C) SFMU et Springer-Verlag France 2012

Un homme de 63 ans a consulté aux urgences suite à des vomissements et un arrêt du transit depuis trois jours. À la prise en charge, il présentait un état de choc et une distension abdominale mais pas de défense. Le scanner abdominal réalisé après remplissage vasculaire révélait une aéroportie (Fig. 1). L'aéroportie se caractérise par des images aériques ramifiées au sein du parenchyme hépatique. Elles ont une distribution sous-capsulaire, l'air étant entrainé par le flux sanguin portal vers la périphérie du foie (contrairement à l'aérobilie). Le traitement et le pronostic de l'aéroportie dépendent essentiellement de la pathologie sousjacente. Si l'infarctus mésentérique a un pronostic sombre, d'autres étiologies peuvent bénéficier d'un traitement médical avec un bon pronostic : maladie de Crohn, diverticulite, pancréatite, ulcère gastrique [1]. Dans notre cas, le patient présentait une occlusion du grêle secondaire à une hernie inguinale étranglée. Il a quitté l'hôpital cinq jours après sa chirurgie.

\section{Référence}

1. Abboud B, El Hachem J, Yazbeck T, et al (2009) Hepatic portal venous gas: physiopathology, etiology, prognosis and treatment. World J Gastroenterol 15:3585-90

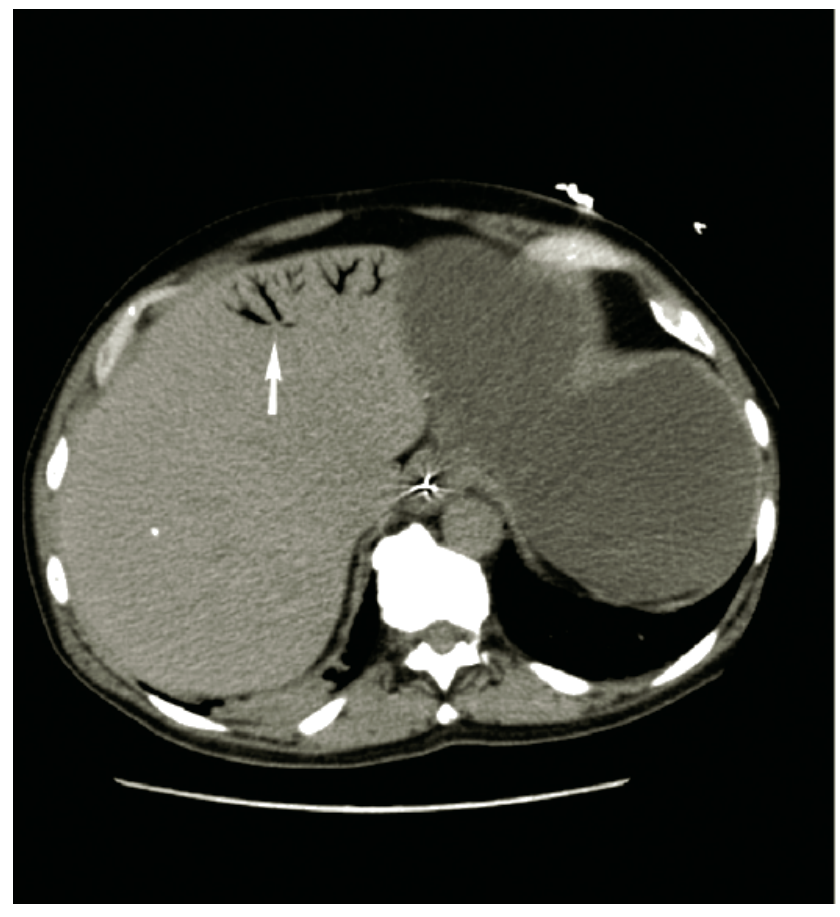

Fig. 1 Images aériques ramifiées du parenchyme hépatique : aéroportie. La topographie sous-capsulaire permet le diagnostic différentiel avec l'aérobilie
M. Tesnière

Services des urgences, Groupe hospitalier mutualiste,

8, rue Docteur Calmette, F- 38000 Grenoble, France

L. Marie $\cdot$ P. Lesage $(\varangle)$

Service d'accueil des urgences,

centre hospitalier de Chambéry, F-73000 Chambéry, France

e-mail : tesniere.marc@yahoo.fr 